\title{
Novel Power Efficient Optical OFDM Based on Hartley Transform for Intensity-Modulated Direct-Detection Systems
}

\author{
Michela Svaluto Moreolo, Raül Muñoz, and Gabriel Junyent
}

\begin{abstract}
We present a novel optical orthogonal frequency division multiplexing (O-OFDM) scheme, suitable for intensity-modulated direct-detection systems, where the modulation/demodulation processing takes advantage of the fast Hartley transform algorithm. Due to the properties of the discrete Hartley transform (DHT), the conventional transmission scheme can be streamlined. We demonstrate that asymmetrically clipping (AC) technique can also be applied to DHT-based OFDM; the signal can be transmitted without the need of a DC bias, resulting in a power-efficient system, not affected by clipping noise. Hermitian symmetry is not required for the input signal. Therefore, this technique supports the double of input symbols compared to both AC and DC-biased O-OFDM, based on standard Fourier processing. The analysis in an additive white Gaussian noise channel shows that the same performance can be achieved by replacing 4, 16, and 64 QAM (quadrature-amplitude modulation) AC optical-OFDM with a simpler system based on DHT, using binary phase-shift keying (BPSK), 4 and 8 PAM (pulse-amplitude modulation), respectively.
\end{abstract}

Index Terms-Asymmetrically clipped optical (ACO) OFDM, discrete Hartley transform (DHT), intensity-modulated direct detection (IM/DD), optical communication, orthogonal frequency-division multiplexing (OFDM).

\section{INTRODUCTION}

$\mathbf{O}$ RTHOGONAL frequency-division multiplexing $(\mathrm{OFDM})$ is a very promising technique, borrowed from broadband wireless and radio communications, for future high-speed large-capacity optical networks, and recently it also represents a novel candidate for optical access networks. As multicarrier transmission technique, OFDM allows transmission of the signal over several lower-rate subchannels. The subcarriers are orthogonal to each other, and their spectra are allowed to overlap. This results in a very high spectral efficiency. Therefore, the use of OFDM in optical networks meets the twofold requirement of mitigating transmission impairments

Manuscript received July 31, 2009; revised October 09, 2009 and December 07, 2009. First published January 26, 2010; current version published March 05,2010 . This work was supported by the Spanish Ministry of Science and Innovation under the Project DORADO (TEC2009-07995) and developed within the Building the Future Optical Network in Europe, a Network of Excellence funded by the European Commission through the 7th Information and Communication Technologies-Framework Programme.

M. Svaluto Moreolo and R. Muñoz are with the Centre Tecnològic de Telecomunicacions de Catalunya, 08860 Barcelona, Spain (e-mail: michela.svaluto@cttc.es).

G. Junyent is with the Universitat Politècnica de Catalunya, 08034 Barcelona, Spain and also with the Centre Tecnologic de Telecomunicacions de Catalunya, 08860 Barcelona, Spain

Digital Object Identifier 10.1109/JLT.2010.2040580 and providing high-data rate transmission. Its resilience to dispersion impairments can reduce the conventional per-span compensation and offer an alternative electronic dispersion compensation method to traditional optical pre- and postcompensation techniques [1], [2]. The high tolerance to chromatic dispersion and polarization mode dispersion allows extending the attainable distance before significant distortion to thousands of kilometers [2]-[9].

The signal processing in the OFDM transmitter/receiver takes advantage of the efficient algorithm of fast Fourier transform (FFT) to implement OFDM modulation/demodulation. On one side, it enables the use of the mature technology and capabilities of DSP; on the other side, it gives a complex and bipolar signal that must be transmitted on an optical link.

To solve this critical issue, alternative solutions have been proposed. Direct detection (DD) and coherent schemes can be used, trading simplicity against increased sensitivity [5]-[13]. DD systems are simpler and can be implemented by using commercial components [6]; no laser is required as local oscillator at the receiver. The simplicity of this cost-effective solution is at expenses of the spectral efficiency, and its effectiveness depends on the system linearity [6], [10]. Coherent detection allows to directly implement the standard RF OFDM scheme, but it is more costly and sensitive to phase noise and frequency offset; the RF-to-optical up-conversion and the optical-to-RF downconversion require narrow linewidth lasers. In this paper, DD is considered. In particular, we provide an alternative OFDM processing for intensity-modulated (IM) optical systems. An OFDM signal to be transmitted on an IM system must be converted into real and positive. To generate real OFDM symbols, the input signal mapped into a complex constellation is forced to have Hermitian symmetry. Adding a DC bias to real signals is an inefficient solution in terms of optical power, to obtain unipolar signals. Usually, the bias value is at least two times the standard deviation of the signal. In presence of high negative picks, the noise due to the clipping at zero level affects the transmission. In 2006, Armstrong and Lowery proposed asymmetrically clipping (AC) as a power-efficient technique to transmit OFDM signals on optical systems, without clipping noise [14]. Only the odd subcarriers are modulated by a signal with Hermitian symmetry.

An alternative optical OFDM scheme can be based on a real trigonometric transform to directly deal with real signal. In this paper, we propose a novel optical OFDM scheme based on the discrete Hartley transform (DHT) for power efficient transmission in IM/DD systems. 


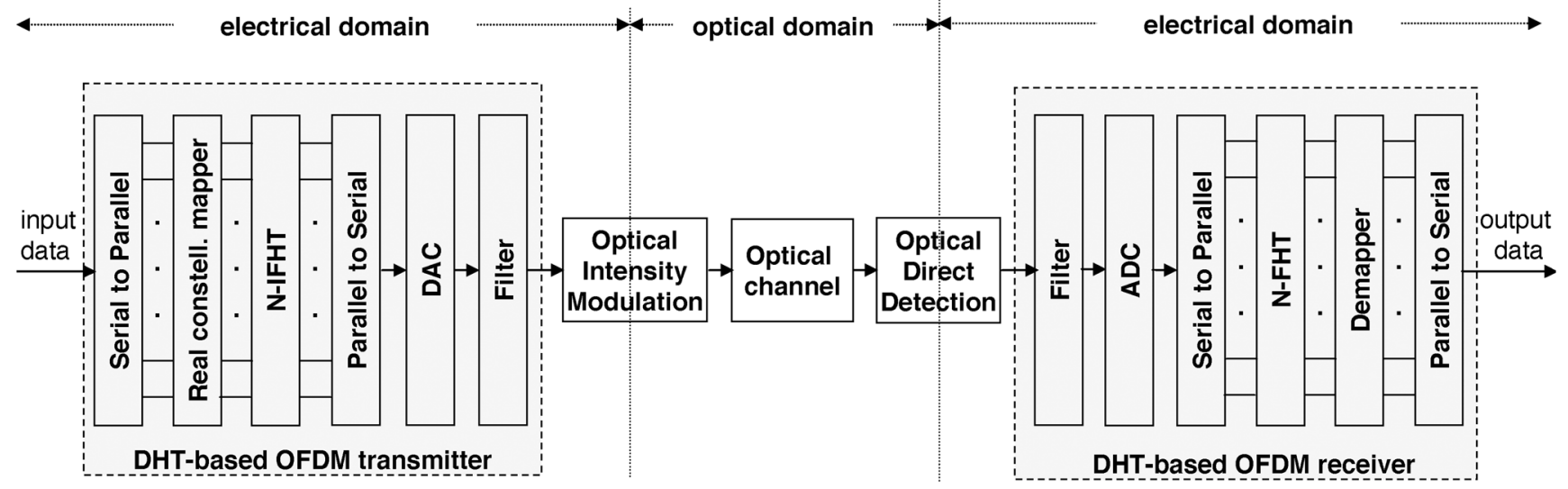

Fig. 1. Block diagram of an IM/DD system, using optical OFDM based on Hartley transform. The input sequence is mapped into a real constellation and OFDM modulated by the $N$-order IFHT. The demodulation uses the direct FHT.

Hartley transform is particularly attractive for the processing of real signals. The direct and inverse transforms are identical, and the Hartley transform of a real signal is real. Fourier transform always implies a complex processing and the phase carries fundamental information, while Hartley transform is a real trigonometric transform. Furthermore, the real and imaginary parts of the discrete Fourier transform (DFT) coincide with the even and the negative odd parts of the DHT, respectively: the transform kernels only differ for the imaginary unit [15]. DFT is used to perform the OFDM modulation, because it can be seen as a bank of modulators, whose narrowband channels have mutually orthogonal subcarriers. Similarly, the mirror-symmetric sub-bands of DHT ensure subcarriers orthogonality, and the spectral behavior enable to carry the data symbols for the parallel processing [16]. Therefore, fast Hartley transform (FHT) can replace FFT algorithm to furnish an alternative OFDM scheme. If the input symbols are real, the inverse FHT (IFHT) gives real OFDM signals. When the OFDM signals are real valued, the multicarrier transmission technique is considered a special case of OFDM. No in-phase and quadrature modulation onto an RF carrier is required and in the literature it is referred as discrete multi-tone modulation (DMT) [17], [18]. Because of the DHT real processing, a simpler transmission system can be achieved, as demonstrated for high-speed wireless communications [19]. We adapt the DHT-based solution proposed in [19] to optical systems. To the best of our knowledge, no demonstration for IM/DD optical OFDM systems based on Hartley transform exists in the literature.

The paper is organized as follows: in Section II, we describe the optical OFDM system based on DHT. In Section III, we demonstrate that the AC technique can be applied to this alternative modulation scheme, without Hermitian symmetry constrain for the input signal. In Section IV, we present the performance of DHT-based AC optical OFDM (ACO-OFDM) in an additive white Gaussian noise (AWGN) channel. We compare it with DC-biased DHT-based OFDM and analyze different real constellations. We also compare our results to the performance of DC-biased and ACO-OFDM systems based on Fourier transform. Finally, in Section V conclusions are drawn.

\section{OPTICAL OFDM SYSTEM BASED ON DHT}

The block diagram of the DHT-based optical OFDM system is depicted in Fig. 1. The IFHT and FHT are used in place of the inverse FFT (IFFT) and FFT, to perform the OFDM modulation and demodulation, respectively. According to the definition of DHT [15], the OFDM symbol is given by

$$
\begin{array}{r}
h(k)=\frac{1}{\sqrt{N}} \sum_{n=0}^{N-1} x(n)[\cos (2 \pi k n / N)+\sin (2 \pi k n / N)] \\
k=0,1,2, \ldots, N-1
\end{array}
$$

where $x(n)$ indicates the symbol sequence and $N$ represents the number of symbols processed in parallel. The DHT kernel is real, and it can also be indicated as [15]

$$
\operatorname{cas}(2 \pi k n / N)=\cos (2 \pi k n / N)+\sin (2 \pi k n / N) .
$$

If a real constellation [e.g., binary phase-shift keying (BPSK), $M$-PAM (pulse-amplitude modulation)] is used for the subcarriers modulation, the OFDM symbol $h(k)$ is real. If the input symbols are complex, as the 16 symbols 4 QAM (quadratureamplitude modulation) of Fig. 2, $h(k)$ is complex. Fig. 2 shows the data blocks at the input and output of a 16-order IFHT, compared to a 16-order IFFT. Both the discrete transforms have a nonzero imaginary part. The IFFT has been evaluated so that the forward and inverse transform has the same normalizing factor $1 / \sqrt{N}$

$$
\begin{aligned}
& f(k)=\frac{1}{\sqrt{N}} \sum_{n=0}^{N-1} x(n) \exp (j 2 \pi k n / N) \\
& \quad k=0,1,2, \ldots, N-1 .
\end{aligned}
$$

In order to obtain real-valued IFFT, the input vector must have Hermitian symmetry. Fig. 3(a) shows the real-valued OFDM symbol at the output of a 32-order IFFT. The information sequence is mapped into 4 QAM symbols; the second-half of the input vector is given by their complex conjugate values [indicated in Fig. 3(a) as QAM*]. The dc and Nyquist frequencies that are $x(0)$ and $x(N / 2)=x(16)$, respectively, are set to zero. 



Fig. 2. From the top, input sequence of 16 symbols 4 QAM; outputs of its discrete 16-order IFFT and 16-order IFHT.

For a given OFDM signal bandwidth, when the number of subcarriers is doubled the carrier spacing decreases accordingly. On the other hand, for a given bit rate, by maintaining the same carrier spacing, if only half of the available subcarriers are used to carry data, the required bandwidth to transmit the same data signal increases accordingly. For a given constellation size, the data rate that can be supported by the double of subcarriers is greater. In DMT systems, only half of the IFFT points are used to process the information data symbols (independent complex values); the second-half is required to process the complex conjugate vector, due to the Hermitian symmetry constrain [18]. When DHT is used, Hermitian symmetry is not required: if the input vector is real, the IFHT is real-valued and the number of subcarriers carrying information symbols (independent real-valued values) coincides with the DHT points, as shown in Fig. 3(b). So that to transmit the same data signal, a lower constellation size (BPSK) is required. In the remainder of the paper, we compare O-OFDM system based on IFHT and real-valued IFFT of order $N$ able to transmit the same data signal.

As shown in Fig. 1, by using the DHT, the input data stream is serial-to-parallel converted and mapped into a real constellation to generate a real OFDM signal. After parallel-to-serial conversion, the real OFDM signal can be processed by one single digital-to-analog converter (DAC). The analog signal is real, but it is still bipolar and must be converted into unipolar to be IM. In the following section, we demonstrate that both AC and DC-biased solutions, adopted in standard optical OFDM, are possible for DHT-based optical OFDM. Adding a DC-bias requires more power and residual clipping noise can affect the transmission. By adopting $\mathrm{AC}$ technique the average optical power is reduced without affecting the transmission.

At the receiver, after DD, the signal is converted to digital by one single analog-to-digital converter (ADC) and the sequence is recovered by DHT processing and demodulating the signal.
As in the standard implementation of OFDM, a cyclic prefix (CP) can be used to mitigate intersymbol interference and intercarrier interference [19]. The choice of prefix length should take into account that this overhead reduces the supported data rate, to cope with the delay introduced by the channel. Generally, the CP is a small fraction of the OFDM symbol, but to be effective should be longer than the delay spread. Mandyam in [20] demonstrates that sinusoidal transforms with a symmetric extension may outperform DFT, when delay spread due to the channel is longer than CP duration. Merched extends the study to the Hartley domain [21]. However, due to the double-side band optical spectrum, ACO-OFDM does not enable to compensate for significant fiber chromatic dispersion [22]. In fact, the intensity modulation generates an OFDM signal on both sides of the optical carrier frequency. DD is more robust to dispersion impairments when combined with optical single-side band modulation than when direct IM is applied [5].

\section{A. Computational Complexity}

We compare the FHT algorithms with optimized algorithms to evaluate the FFT of real-valued sequences. When FFT exploits the Hermitian symmetry property of the transform, FHT algorithms require about the same number of multiplications but more additions [23]-[25]. Nevertheless, additional resources must be used for calculating the complex conjugate vector to deal with real-valued FFT. In the case of radix-2 algorithm, reported in [25], for both the decimation-in-time and decimation-in-frequency, the number of multiplications required by the DHT is $N \log _{2} N-3 N+4$ and the number of additions is $\left(3 N \log _{2} N-3 N+4\right) / 2$, with $N$ the transform order. The FHT-based algorithm has the same number of multiplications and $N-2$ more additions than the corresponding FFT algorithm optimized for a real input vector. Similarly, the number of additions required by FHT slightly exceeds the ones required by FFT of a real-valued sequence for radix-4, split radix, prime factor, and Winograd transform algorithms, as demonstrated in 1985 by Sorensen et al. [25]. In 1986, Duhamel and Vetterli proposed the fastest algorithm implementing the DHT. Its improved version requires only two more additions than the FFT algorithms for real-valued signal with minimum arithmetic complexity [26], [27]. This improvement increases the computational speed in DSP devices. The minimum number of multiplications for both FFT and FHT is $P=\left(N \log _{2} N-3 N+4\right) / 2$, while the number of additions is $A^{\mathrm{FFT}}=\left(3 N \log _{2} N-5 N\right) / 2+4$ and $A^{\mathrm{FHT}}=\left(3 N \log _{2} N-5 N\right) / 2+4$, respectively. The number of total operations required for 32-order transforms is indicated in Fig. 3, and it is also shown where additional computational resources are needed.

Moreover, although the FFT and IFFT are very similar, the same FHT routine can be applied to calculate the forward and inverse transforms, because DHT is self-inverse. Therefore, the same DSP device can be used for modulation and demodulation. To change from the forward to the IFFT transform, an additional control is required to reverse the sign of the imaginary unit in the transform kernel. 
(a)

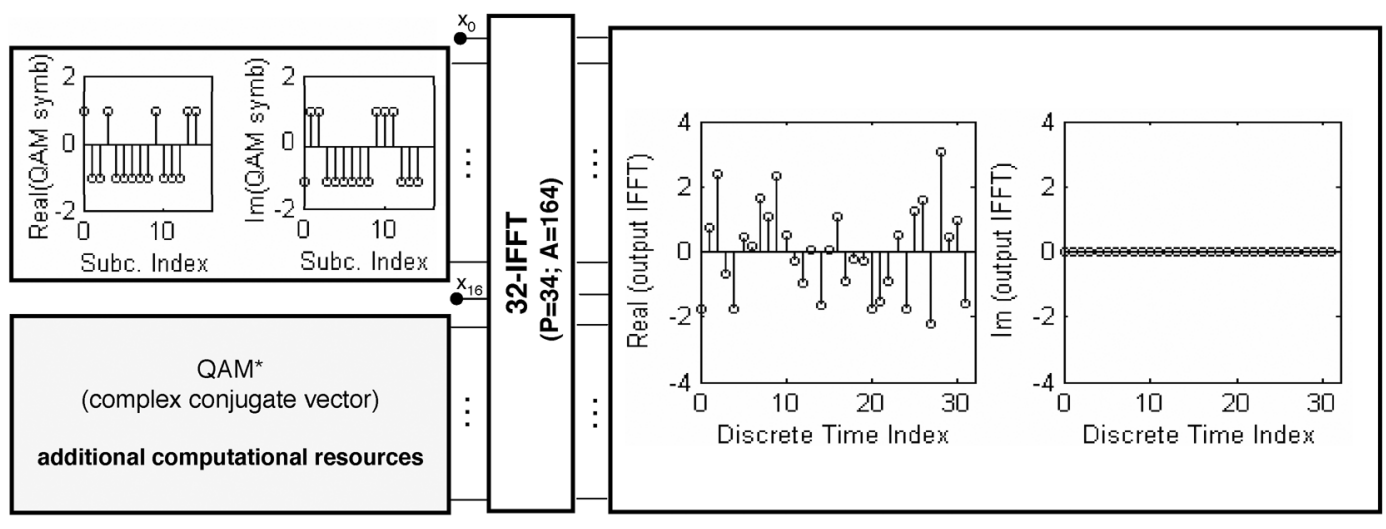

(b)
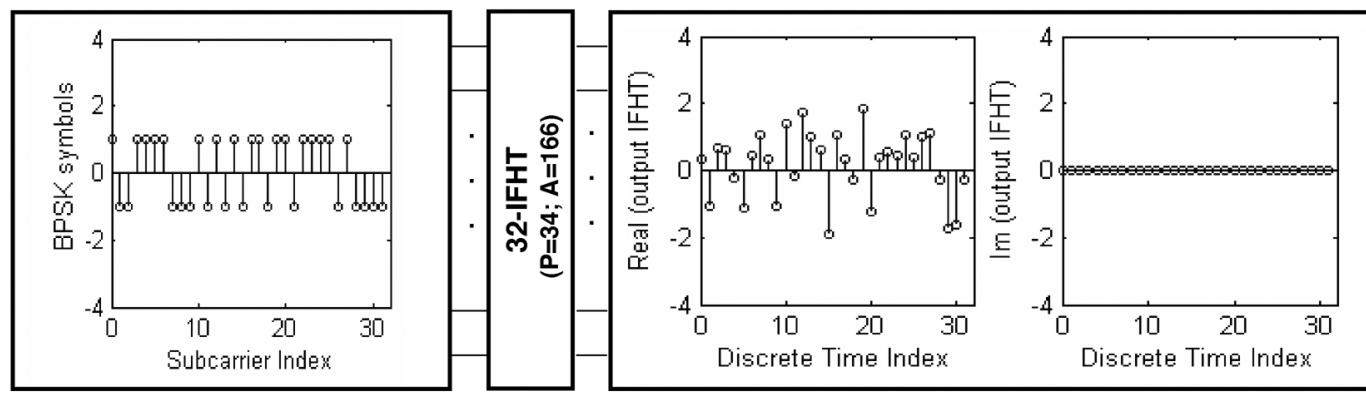

Fig. 3. (a) Real-valued discrete outputs of a 32-order IFFT. The input sequence has Hermitian symmetry, and it is given by the half-length vector of 4 QAM symbols and the corresponding complex conjugate vector. The dc and Nyquist frequencies are set to zero. (b) Real-valued discrete DHT-based OFDM symbol, evaluated as the 32-order IFHT of a BPSK vector of length 32. The number of multiplications (P) and additions (A) reported in the figure have been evaluated according to the FFT and FHT algorithms with the minimum arithmetic complexity [27].

\section{Asymmetrically CliPPed DHT-BASED O-OFDM}

In this section, we demonstrate that $\mathrm{AC}$ technique can also be applied to OFDM signals generated by using the Hartley transform. With a suitable choice of the subcarriers to be modulated, the DHT-based OFDM signal can be clipped at zero level and correctly recovered without clipping noise.

In fact, as for DFT, also for DHT it is easy to demonstrate that for $n$ odd

$$
h(n, k+N / 2)=-h(n, k) .
$$

Therefore, if only the odd subcarriers are nonzero, the DHTOFDM symbol is redundant, as shown in Fig. 4(a), and can be clipped without losing information. The nonredundant OFDM symbol is shown in Fig. 4(b); it is obtained by clipping the IDHT evaluated for a BPSK sequence of length $N=64$. The sequence $x(n)$ can be recovered by simply performing the Hartley transform of the IDHT-OFDM symbol

$$
x(n)=\frac{1}{\sqrt{N}} \sum_{k=0}^{N-1} h(k)[\cos (2 \pi k n / N)+\sin (2 \pi k n / N)] .
$$

By making explicit the symbol elements in the summation, it can be rewritten in the following form

$$
\begin{aligned}
x(n)=\frac{1}{\sqrt{N}} \sum_{k=0}^{N / 2-1} h(k) \operatorname{cas}(2 \pi k n / N) \\
+h(k+N / 2) \operatorname{cas}(2 \pi k n / N+\pi n) .
\end{aligned}
$$

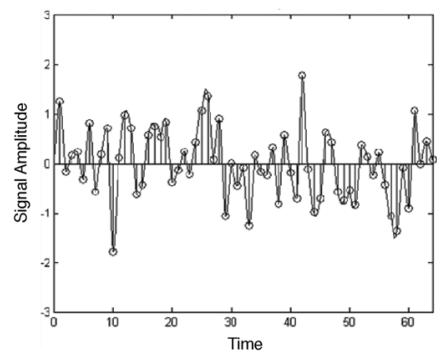

(a)

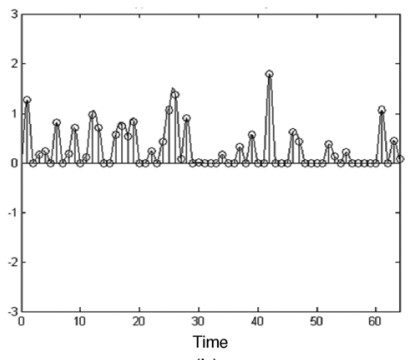

(b)
Fig. 4. (a) BPSK signal OFDM modulated with a 64-order DHT, in digital $h(k)$ and analog $h(t)$ form; (b) corresponding clipped DHT-OFDM symbol.

Stated (4) and according to the assumption that only the odd subcarriers are nonzero

$$
\begin{gathered}
x(n)=\frac{2}{\sqrt{N}} \sum_{\substack{k=0 \\
h(k)>0}}^{N / 2-1} h(k) \operatorname{cas}(2 \pi k n / N) \\
+\frac{2}{\sqrt{N}} \sum_{\substack{k=0 \\
h(k)<0}}^{N / 2-1} h(k) \operatorname{cas}(2 \pi k n / N)
\end{gathered}
$$

where the summation in (6) has been split in two, depending on the sign of $h(k)$. For clipped signal $h_{c}(k)$

$$
h_{c}(k)= \begin{cases}h(k) & h(k)>0 \\ 0 & h(k) \leq 0\end{cases}
$$



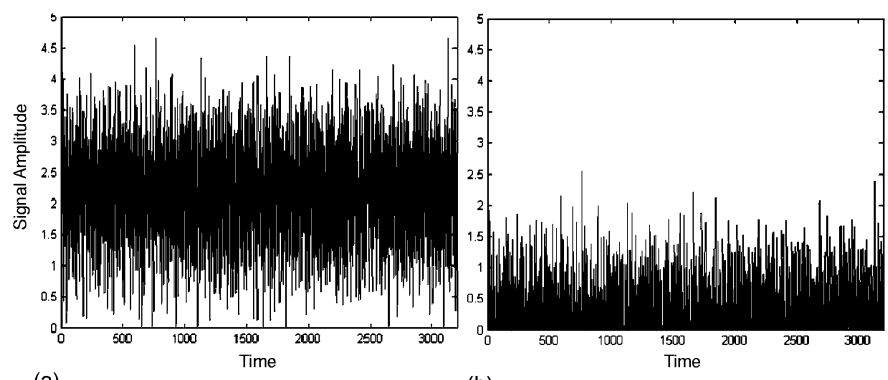

(a)

(b)

Fig. 5. Analog signal representing 50 OFDM symbols mapped into BPSK constellation and modulated with 64-order DHT (a) DC-biased and (b) clipped.

only one of the two terms of (6) is nonzero, depending on the sign of $h(k)$; therefore, the recovered symbol sequence will be

$$
r(n)=\frac{1}{\sqrt{N}} \sum_{k=0}^{N-1} h_{c}(k) \operatorname{cas}(2 \pi k n / N)=\frac{x(n)}{2} .
$$

This means that, as in ACO-OFDM based on FFT [14], the symbol sequence can be recovered from the odd subcarriers, and the constellation points have the half of the original values. All the clipping noise falls into the even subcarriers that can be easily discarded.

As clipping is a memoryless nonlinearity, the attenuation of the input sequence and the presence of additive noise directly follow the Bussgang's theorem. It can be applied because $h(k)$ can be assumed to have a Gaussian distribution for $N \geq 64$. Therefore, the same analysis of clipping reported in [14] can be considered valid also for the DHT-OFDM signal.

\section{A. Simulation Results}

To prove the correctness of the theoretical approach, we simulate the transmission of 50 OFDM symbols in a back-to-back system based on DHT processing. The input sequence is mapped into BPSK constellation points and modulated by a $N=64$ DHT. Only the odd subcarriers are modulated; therefore, in an ACO-OFDM system based on $\mathrm{N}$-order DHT, only $N / 2$ data symbols can be transmitted. However, as shown in Fig. 5, the optical power, which is proportional to the electrical OFDM signal, is substantially reduced, compared to the DC-biased signal, for which all the subcarriers can be modulated. The bias value should be at least two times the signal standard deviation. The choice of a fixed bias value trades power efficiency and additional noise. In fact, in DC-biased optical systems the reduction of clipping noise is at expenses of power consumption.

At the receiver side, the DHT of the clipped signal is performed to recover the BPSK sequence. Fig. 6 shows the received constellation: the symbols, with half of the original value, can be recovered from the odd subcarriers, and the even subcarriers represent the noisy component of the signal.

\section{B. Comparison Between DHT-Based and DFT-Based ACO-OFDM Transmission Systems}

An OFDM system based on $N$-order DHT can transmit $N$ independent real-valued symbols. In DFT-based OFDM, the

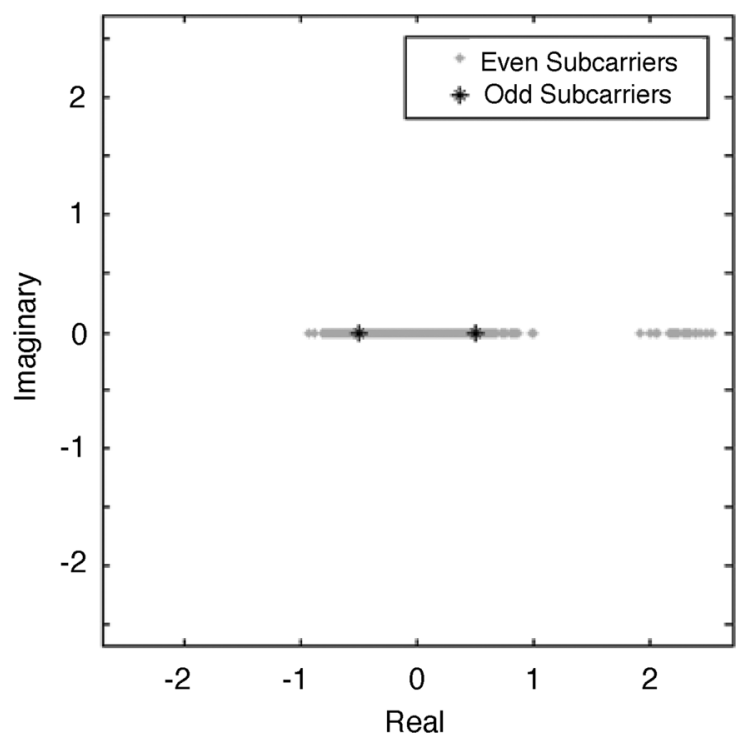

Fig. 6. Received constellation in a DHT-based OFDM system, using AC. The recovered BPSK signal with the half of the original values is carried by the odd subcarriers and the clipping noise falls into the even subcarriers.

number of independent symbols that can be transmitted with a $N$-order DFT is reduced to $N / 2$, due to the Hermitian symmetry constrain on the input sequence.

Consider transmitting a certain bit rate over $N=64$ subcarriers. ACO-OFDM requires half the carriers to be unused. At each parallel processing, 32 bits are mapped into 4 QAM constellation and transmitted over $N / 4=16$ odd subcarriers. The remaining 16 are used to carry the complex conjugate vector. By using Hartley transform, all the $N / 2=32$ carriers carry information symbols. The input bits are mapped into a simpler BPSK constellation to transmit the same bit rate.

Fig. 7 shows the transmission of the same bit sequence by using either an IFHT or an IFFT of order $N=64$ and the resulting real-valued OFDM signals. The 32 even-indexed subcarriers are set to zero in order to avoid signal distortion due to the clipping noise. According to [27], $P=98$ multiplications and $A=422$ additions are required for the FHT. That is only two more additions compared to the real-valued FFT algorithm with the minimum arithmetic complexity.

\section{PERformance AnAlysis}

We analyze the performance of the proposed IM/DD optical OFDM communication systems based on DHT, in an AWGN channel. We add a Gaussian noise source in the electrical domain, after DD, to the system represented in Fig. 1. In order to compare the performance of the proposed system, with AC and DC-biased O-OFDM systems based on FFT presented by other authors [28], [29], we consider the same assumptions. The comparison that we propose is between DMT systems based on $N$-order DHT, and DFT able to transmit the same data signal per OFDM symbol, i.e., the same information bit sequence per parallel processing. We assume that the impulse response of the optical channel is unitary, and we do not consider the CP. For the analysis of DC-biased solution, we evaluate the performance 


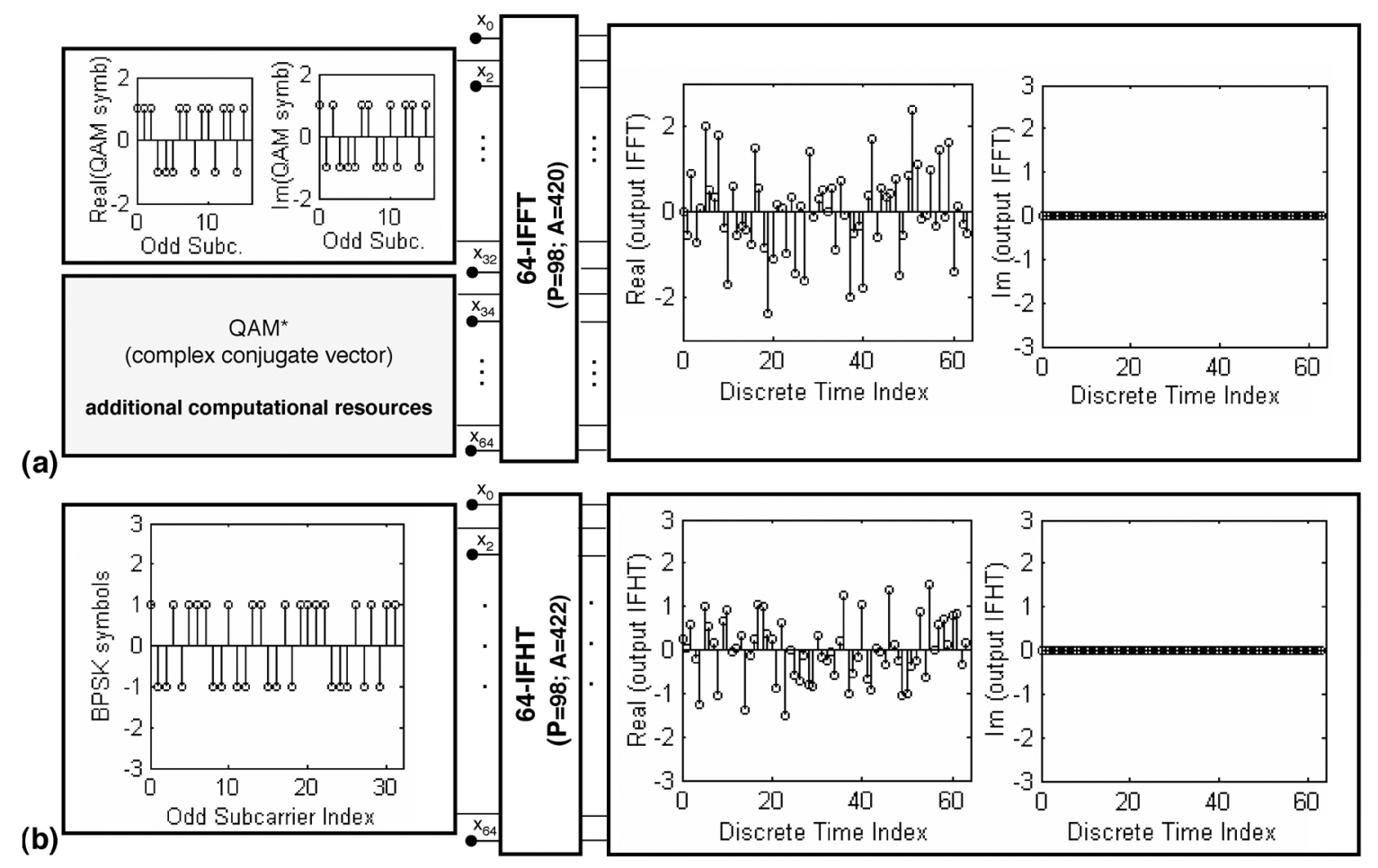

Fig. 7. 16 symbols 4 QAM and 32 symbols BPSK, representing the same input sequence of 32 bits. The corresponding real-valued discrete OFDM symbols using (a) a 64-order IFFT and (b) a 64-order IFHT have been evaluated modulating only the odd subcarriers. The even subcarriers are set to zero. The second-half of IFFT odd subcarriers are modulated by the complex conjugate values of the 16 symbols 4 QAM. The number of multiplications (P) and additions (A) reported in the figure have been evaluated according to [27].

for bias values of 7 and $13 \mathrm{~dB}$, assuming the same bias definition of [29]

$$
B_{\mathrm{dc}}=k \sqrt{E\left\{h^{2}(t)\right\}}
$$

and considering its value in $\mathrm{dB}$ as $10 \log _{10}\left(k^{2}+1\right) ; E\left\{h^{2}(t)\right\}$ is the signal variance. We compare the proposed AC DHT-based $\mathrm{O}-\mathrm{OFDM}$ to the DC-biased solution, considering a BPSK modulation format for the input data sequence. Fig. 8 reports the bit error rate (BER) as a function of the bit electrical energy normalized to the noise power spectral density $\left(E_{b(\text { elec })} / N_{0}\right)$.

Due to the Bussgang's theorem and the considerations reported in [14], [28], and [29], the relation between electrical and optical power can be easily derived for both AC and DC-biased O-OFDM systems. Therefore, we refer to the electrical energy for this analysis and not to the optical power. The electrical-tooptical conversion can be obtained by using formulas (4) and (5) in [29]. To give an example, we calculate the optical power for the DHT-based DCO-OFDM, using BPSK modulation and a DC-bias of $7 \mathrm{~dB}$. The corresponding value of $k$ is 2 , according to (10). The normalized optical power per transmitted bit for a BER of $10^{-3}$ is $E_{b(\text { opt })} / N_{0}=10 \log _{10}\left(k^{2} /\left(k^{2}+1\right)\right)+14 \mathrm{~dB}=$ $13 \mathrm{~dB}$, being the normalized bit electrical energy $E_{b(\text { elec })} / N_{0}=$ $14 \mathrm{~dB}$ (see Fig. 8). By using the same modulation format, with ACO-OFDM based on DHT the normalized optical power per transmitted bit is $E_{b(\mathrm{opt})} / N_{0}=10 \log _{10}(1 / \pi)+9.8 \mathrm{~dB}=4.8$ $\mathrm{dB}$. The same result has been reported in [29] for an ACOOFDM based on FFT using 4 QAM.

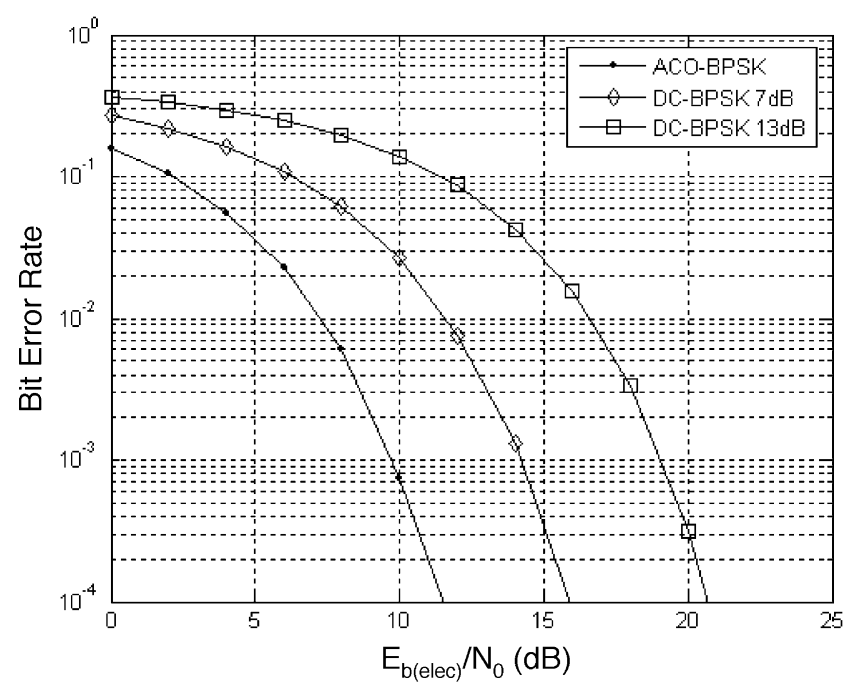

Fig. 8. BER versus normalized bit electrical energy $\left(E_{b(\text { elec })} / N_{0}\right)$ for BPSK ACO and DC-biased OFDM based on DHT in AWGN channel.

The power efficiency of AC technique is superior to dc-biased solution also for optical DHT-based OFDM, as shown in Fig. 8. However, due to the $\mathrm{AC}$, which reduces the recovered symbols to half of the original values (see Fig. 6), in ACO-OFDM $3 \mathrm{~dB}$ more power is required compared to a bipolar system, using the same constellation [29]. Only half of the power is used for the odd subcarriers, the rest is converted into clipping noise carried by the even subcarriers. For this reason, in the ACO-OFDM system of Fig. 8 , the required $E_{b(\text { elec })} / N_{0}$ is $9.8 \mathrm{~dB}$, instead of 
TABLE I

COMPARISON BETWEEN DHT-BASED AND DFT-BASED O-OFDM FOR IM/DD SYSTEMS

\begin{tabular}{|c|c|c|}
\hline \multirow{2}{*}{$\begin{array}{c}\text { Optical OFDM } \\
\text { type }\end{array}$} & N-order DHT-based & $\mathrm{N}$-order DFT-based \\
\hline & DC-biased & DC-biased \\
\hline $\begin{array}{l}\text { Supported } \\
\text { constellation }\end{array}$ & $\begin{array}{c}\text { Real } \\
\text { (BPSK, M-PAM) }\end{array}$ & $\begin{array}{l}\text { Complex } \\
\text { (m-QAM) }\end{array}$ \\
\hline Constell. size & $\mathrm{M}=2^{\log _{4} L}$ & $\mathrm{~m}=L$ \\
\hline Hermitian symmetry & NOT required & Required \\
\hline $\begin{array}{l}\text { Computational } \\
\text { complexity [27] }\end{array}$ & $\begin{array}{c}\mathrm{P}=\left(\mathrm{N} \log _{2} \mathrm{~N}-3 \mathrm{~N}+4\right) / 2 \\
\mathrm{~A}=\left(3 \mathrm{~N} \log _{2} \mathrm{~N}-5 \mathrm{~N}\right) / 2+6 \\
\text { Self-inverse } \\
\mathrm{NO} \text { add. resources }\end{array}$ & $\begin{array}{c}\mathrm{P}=\left(\mathrm{N} \log _{2} \mathrm{~N}-3 \mathrm{~N}+4\right) / 2 \\
\mathrm{~A}=\left(3 \mathrm{~N} \log _{2} \mathrm{~N}-5 \mathrm{~N}\right) / 2+4 \\
\text { NOT self-inverse } \\
\text { Resources for QAM }{ }^{*}\end{array}$ \\
\hline Subc. carrying info & $\mathrm{N} / 2$ & $\mathrm{~N} / 2$ \\
\hline
\end{tabular}

the $6.8 \mathrm{~dB}$ required by a BPSK system that is not forced to be unipolar. In the case of DC-biased O-OFDM, all the subcarriers are used to modulate the input symbols, while in the case of $\mathrm{AC}$, only the odd subcarriers are used for modulation. There is a tradeoff between bandwidth efficiency and power efficiency. In fact, Fig. 8 shows that to obtain a BER of $10^{-3}$, DC-biased OFDM with a bias of two times the standard deviation [i.e., 7 $\mathrm{dB}$, according to (10)], requires a normalized bit energy value about $4 \mathrm{~dB}$ greater than the $E_{b(\mathrm{elec})} / N_{0}$ of ACO-OFDM. In our performance evaluation, we take into account the additional noise due to the clipping at zero level of DC-biased signal. If the bias value is larger, the additional noise due to the clipping of residual negative peaks decreases. This is at expenses of the electrical power, as it can be seen for the BER curve of Fig. 8, representing the performance of a DHT-based optical OFDM system using $13 \mathrm{~dB}$ as bias value.

As summarized in Table I, the independent constellation symbols supported by an $N$-order FFT-based AC or DC-biased O-OFDM are $N / 4$ and $N / 2$, respectively. For DHT-based are $N / 2$ and $N$, since the double of constellation symbols can be allocated. Therefore, in order to compare O-OFDM systems transmitting the same signal at the same bit rate, the input sequence of the DHT-based scheme is mapped into a real $M$-ary constellation that requires a lower value for $M$.

We first compare the performance of AC DHT-based systems using a 2 levels modulation (BPSK) with the ACO-OFDM based on FFT analyzed in [28], where the modulation is a 4 QAM. We observe that the same performance can be achieved with a simpler scheme, using the DHT real processing. Similarly, the same BER curves can be obtained with a DC-biased DHTbased O-OFDM system, using BPSK or a 4 QAM DC-biased system based on FFT of the same order, transmitting the same data sequence. Again, we compare our results with the curves in [29], observing good agreement.

To benefit of the real processing offered by Hartley transform, only real constellation can be supported. We analyze the performance for 4 and 8 PAM. The BER curves are plotted in Fig. 9. The same performance of FFT-based ACO-OFDM using 16 and 64 QAM (see [29, Fig. 1]) can be achieved with 4 and 8 PAM DHT-based ACO-OFDM. The same data sequence at the same bit rate is transmitted with a simpler system, which does not require Hermitian symmetry for the input signal. In an IM/DD optical OFDM system, both FFT and FHT of the same order $N$ can be used to transmit at the same bit rate. However, if $L$ is the QAM constellation size, to achieve the same

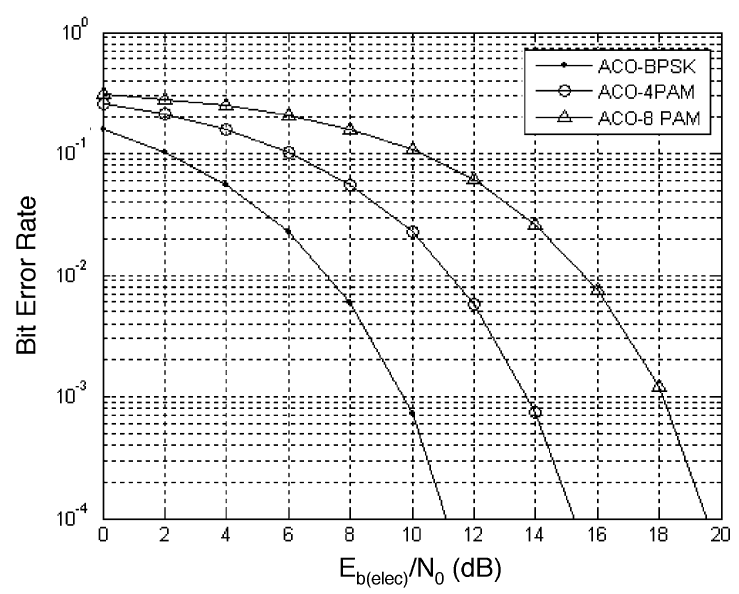

Fig. 9. BER versus normalized bit electrical energy $\left(E_{b(\text { elec })} / N_{0}\right)$ for BPSK, 4 PAM, and 8 PAM ACO-OFDM based on DHT in AWGN channel.

performance, the size of the real constellation for the DHT is $M=2^{\log _{2}(L) / 2}=2^{\log _{4} L}$.

\section{CONCLUSION}

This paper provides a novel optical OFDM scheme, where the real processing of Hartley transform replaces the Fourier processing. Compared to FFT algorithms for real-valued sequence, FHT only requires few more additions. Moreover, the direct and inverse transforms are equal, so that the same fast algorithm can be applied and the same DSP is required for the OFDM modulation and demodulation. If a real constellation is used, the DHT-OFDM symbols are real. One single DAC and one single $\mathrm{ADC}$ are required at the transmitter and receiver, as in DMT systems.

We have demonstrated that DHT-based OFDM signals can be asymmetrically clipped without the need of Hermitian symmetry constrain for the input vector. If only the odd subcarriers are modulated, the signal can be correctly recovered and all the clipping noise falls into the even subcarriers. Moreover, the computational time required to evaluate the complex conjugate of the input constellation symbols vector can be saved. Therefore, the proposed optical OFDM system furnishes a simplified scheme with the same power efficiency of FFT-based ACO-OFDM, resulting suitable for IM/DD optical systems.

The analyzed performance shows that the same BER curves, obtained with FFT-based O-OFDM, can be obtained with the proposed O-OFDM scheme based on DHT. With a DHT of the same order and using a real constellation with lower size, the same data sequence at the same bit rate can be transmitted, adopting either DC-biased or ACO solutions.

\section{ACKNOWLEDGMENT}

The authors would like to thank the anonymous reviewers for their helpful comments and suggestions that have improved the quality of this paper.

\section{REFERENCES}

[1] A. J. Lowery, "Optical OFDM," in Proc. CLEO/QELS 2008, May, pp. $1-2$. 
[2] A. J. Lowery, L. B. Du, and J. Armstrong, "Performance of optical OFDM in ultralong-haul WDM lightwave systems," J. Lightw. Technol., vol. 25, no. 1, pp. 131-138, Jan. 2008.

[3] A. J. Lowery and J. Armstrong, "Orthogonal-frequency-division multiplexing for dispersion compensation of long haul optical systems," Opt. Exp., vol. 14, pp. 2079-2084, 2006.

[4] A. J. Lowery and J. Armstrong, "Dispersion compensation in long haul transmission systems-An orthogonal approach," presented at the Conf. Optical Internet 2007, Melbourne, Australia, Jun.

[5] B. J. C. Schmidt, A. J. Lowery, and J. Armstrong, "Experimental demonstration of $20 \mathrm{Gbit} / \mathrm{s}$ direct-detection optical OFDM and 12 Gbit/s with a colorless transmitter," presented at the Optical Fiber Communication Conf. 2007, Anaheim, CA, Mar. , Paper PDP 18.

[6] B. J. C. Schmidt, A. J. Lowery, and J. Armstrong, "Experimental demonstration of electronic dispersion compensation for long-haul transmission using direct-detection optical OFDM," J. Lightw. Technol., vol. 26, no. 1, pp. 196-203, Jan. 2008.

[7] S. L. Jansen, I. Morita, T. C. W. Schenk, N. Takeda, and H. Tanaka, "Coherent optical 25.8-Gb/s OFDM transmission over 4160-km SSMF," J. Lightw. Technol., vol. 26, no. 1, pp. 6-15, Jan. 2008.

[8] S. L. Jansen, I. Morita, T. C. W. Schenk, D. van den Borne, and H. Tanaka, "Optical OFDM-A candidate for future long-haul optical transmission systems," in Proc. Opt. Fiber Commun. Conf., 2008, pp. 1-3, Paper OMU3.

[9] S. L. Jansen, I. Morita, T. C. W. Schenk, and H. Tanaka, "121.9-Gb/s PMD-OFDM transmission with $2-\mathrm{b} / \mathrm{s} / \mathrm{Hz}$ spectral efficiency over 1000-km of SSMF," J. Lightw. Technol., vol. 27, no. 3, pp. 177-188, Feb. 2009.

[10] J. Armstrong, "OFDM for optical communications," J. Lightw. Technol., vol. 27, no. 3, pp. 189-204, Feb. 2009.

[11] W. Shieh and C. Athaudage, "Coherent optical orthogonal frequency division multiplexing," Electron. Lett., vol. 42, no. 10, pp. 587-589, May 2006.

[12] W. Shieh, H. Bao, and Y. Tang, "Coherent optical OFDM: Theory and design,” Opt. Exp., vol. 16, pp. 841-859, Jan. 2008.

[13] Q. Yang, Y. Tang, Y. Ma, and W. Shieh, "Experimental demonstration and numerical simulation of $107-\mathrm{Gb} / \mathrm{s}$ high spectral efficiency coherent optical OFDM," J. Lightw. Technol., vol. 27, no. 3, pp. 168-176, Feb. 2009.

[14] J. Armstrong and A. J. Lowery, "Power efficient optical OFDM," Electron. Lett., vol. 46, no. 6, pp. 370-372, 2006.

[15] R. N. Bracewell, "Discrete Hartley transform," J. Opt. Soc. Amer., vol. 73, pp. 1832-1835, Dec. 1983.

[16] M. S. Moreolo, V. Sacchieri, and G. Cincotti, "Signal processing based on trigonometric transforms for high-speed optical networks," presented at the Int. Conf. Transparent Optical Networks (ICTON 2009), Island of São Miguel, Azores, Portugal, 28 Jun.-2 Jul. .

[17] S. C. J. Lee, F. Breyer, S. Randel, M. Schuster, J. Zeng, F. Huijskens, H. P. A. van den Boom, A. M. J. Koonen, and N. Hanik, "24-Gb/s transmission over $730 \mathrm{~m}$ of multimode fiber by direct modulation of an 850-nm VCSEL using discrete multi-tone modulation," presented at the Optical Fiber Communication Conf. 2007, Anaheim, CA, Mar. 2007, PDP 6.

[18] S. C. J. Lee, F. Breyer, S. Randel, H. P. A. Van Den Boom, and A. M. J. Koonen, "High-speed transmission over multimode fiber using discrete multitone modulation," J. Opt. Netw., vol. 7, pp. 183-196, Feb. 2008.

[19] D. Wang, D. Liu, F. Liu, and G. Yue, "A novel DHT-based ultra-wideband system," in Proc. ISCIT 2005, Jun. 2004, vol. 50, pp. 172-184.

[20] G. D. Mandyam, "Sinusidal transforms in OFDM systems," IEEE Trans. Broadcast., vol. 50, no. 2, pp. 172-184, Jun. 2004.

[21] R. Merched, "On OFDM and single-carrier frequency-domain systems based on trigonometric transforms," IEEE Signal Process. Lett., vol. 13, no. 8, pp. 473-476, Aug. 2006.

[22] A. J. Lowery and J. Armstrong, "Comparison of power-efficientoptical orthogonal frequency division multiplexing transmission methods," presented at the Conf. Optical Fibre Technology/Australian Optical Society, Melbourne, Australia, Jul. 2006.

[23] R. N. Bracewell, "The fast Hartley transform," Proc. IEEE, vol. 72, no. 8, pp. 1010-1018, Aug. 1984.

[24] H. S. Hou, "The fast Hartley transform algorithm," IEEE Trans. Comput., vol. C-36, no. 2, pp. 147-156, Feb. 1987.

[25] H. V. Sorensen, D. L. Jones, C. S. Burrus, and M. T. Heideman, "On computing the discrete Hartley transform," IEEE Trans. Acoust., Speech, Signal Process., vol. ASSP-33, no. 5, pp. 1231-1238, Oct. 1985.
[26] P. Duhamel and M. Vetterli, "Cyclic convolution of real sequences: Hartley versus Fouroer and new schemes," in Proc. Int Conf. Acoust., Speech, Signal Process. (ICASSP 1986), Tokyo, pp. 229-232.

[27] P. Duhamel and M. Vetterli, "Improved Fourier and Hartley transform algorithms: Application to cyclic convolution of real data," IEEE Trans. Acoust., Speech, Signal Process., vol. ASSP-35, no. 6, pp. 818-824, Jun. 1987.

[28] J. Armstrong, B. J. C. Schmidt, D. Kalra, H. A. Suraweera, and A. J. Lowery, "Performance of asymmetrically clipped optical OFDM in AWGN for an intensity modulated direct detection system," in Proc. IEEE Global Telecommun. Conf., 2006, pp. 1-5.

[29] J. Armstrong and B. J. C. Schmidt, "Comparison of asymmetrically clipped optical OFDM and DC-biased optical OFDM in AWGN," IEEE Commun. Lett., vol. 12, no. 5, pp. 343-345, May 2008.



Michela Svaluto Moreolo received the M.Sc. degree (with honors) in electronics engineering and the $\mathrm{Ph} . \mathrm{D}$. degree in telecommunications engineering from University Roma Tre, Rome, Italy, in 2003 and 2007, respectively.

From January 2006 to July 2006, she was a Visiting Researcher at the Institute of Semiconductor and Solid State Physics, Johannes Kepler University, Linz, Austria. From January 2007 to December 2008, she was a Postdoctoral Researcher at the Applied Electronics Department of University Roma Tre. She is currently a Research Associate at the Optical Networking Area of the Centre Tecnològic de Telecomunicacions de Catalunya, Barcelona, Spain. Her research interests are in optical signal processing and advanced transmission technologies for high-speed optical networks, including optical OFDM, multiplexing, and coding techniques.

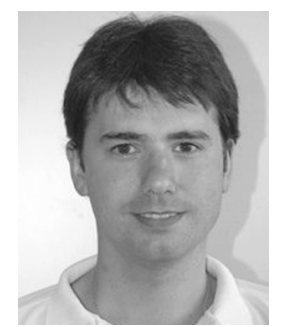

Raül Muñoz graduated in telecommunications engineering, in 2001, and received the Ph.D. degree in telecommunications, in 2005, both from the Universitat Politécnica de Catalunya (UPC), Barcelona, Spain.

$\mathrm{He}$ is currently Senior Research Associate and Coordinator of the Optical Networking Area of the Centre Tecnològic de Telecomunicacions de Catalunya (CTTC), Barcelona, Spain. Since 2000, he has participated in more than $20 \mathrm{R} \& \mathrm{D}$ and technology transfer projects (FP7 IP STRONGEST and NOE BONE, FP6 IP NOBEL and NOBEL2, CELTIC 100GET, etc). He has also led the Spanish projects DORADO, RESPLANDOR, and GAUDI. $\mathrm{He}$ is the author or coauthor of more than 50 papers published in various journals and international conference papers. He has also been a Coordinator of CTTC ADRENALINE Testbed. His research interests include generalized multiprotocol label switching architecture, protocols, and traffic engineering algorithms (provisioning, protection, and restoration strategies) for highly dynamic optical transport networks.

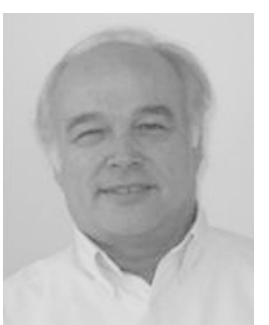

Gabriel Junyent graduated in telecommunication engineering from the Universidad Politécnica de Madrid, Madrid, Spain, in 1973, and received the $\mathrm{Ph} . \mathrm{D}$. degree in communications from the Universitat Politècnica de Catalunya (UPC), Barcelona, Spain, in 1979.

From 1973 to 1989, he was a Teaching Assistant and Associate Professor at the UPC, where he has been a Full Professor since 1989. In the last 15 years he has participated in more than 30 national and international $\mathrm{R} \& \mathrm{D}$ projects. He is the author or coauthor of more than 30 journal papers and book chapters and 100 conference papers. 